Effects of Cariprazine on Cognition in Patients With Bipolar Mania or Mixed States: Post Hoc Analysis From 3 Randomized, Controlled Phase III Studies

\author{
Roger S. Mcintyre, $M D^{1}$, Eduard Vieta, $M D^{2}$, \\ Willie Earley, $\mathrm{MD}^{3}$ and Mehul Patel, PharmD ${ }^{3}$
}

${ }^{1}$ University Health Network, Toronto, ON, Canada, ${ }^{2}$ University of Barcelona, Barcelona, Spain, and ${ }^{3} \mathrm{AbbVie}$, Madison, NJ, USA

Presenting Author: Roger S. McIntyre
Conclusion. In patients with manic or mixed episodes associated with bipolar I disorder, cariprazine versus placebo was effective in improving cognitive symptoms in the overall patient population as well as in patients with baseline cognitive symptoms. In addition, cariprazine versus placebo also demonstrated efficacy in improving manic symptoms in patients with baseline cognitive symptoms. These results suggest that cariprazine may provide benefits for the treatment of cognitive symptoms in patients with bipolar I mania. Funding. AbbVie Inc.

\title{
Evaluation of MADRS Severity Thresholds in Patients With Bipolar Depression
}

\begin{abstract}
Introduction. Cariprazine, a dopamine D3-preferring D3/D2 and serotonin 5-HT1A receptor partial agonist, is approved for the treatment of schizophrenia and for depressive, manic, or mixed episodes associated with bipolar I disorder. Previous post hoc analyses have demonstrated that cariprazine was effective versus placebo for improving cognitive symptoms in patients with schizophrenia or bipolar depression. This post hoc analysis evaluated the effects of cariprazine on cognitive symptoms in patients with acute manic or mixed bipolar episodes.

Methods. Data from 3 phase II/III, randomized, double-blind, placebo-controlled studies in patients with manic or mixed episodes associated with bipolar I disorder (NCT00488618, NCT01058096, NCT01058668) were pooled and analyzed. Patients were randomized to placebo or flexibly dosed cariprazine (3-12 mg/d, 3-6 mg/d, or 6-12 mg/d [1 study only]) for 3 weeks of double-blind treatment; all dose groups were combined for the pooled analysis. Cognitive symptoms were assessed using the Positive and Negative Syndrome Scale (PANSS) Cognitive subscale (sum of PANSS items P2, N5, N7, G10, G11); a score of 15 or greater at baseline indicated the presence of cognitive symptoms. Mean changes from baseline to week 3 in PANSS cognitive subscale/item scores and Young Mania Rating Scale (YMRS) total score were evaluated in the overall intent-to-treat (ITT) population and in the subgroup of patients with baseline cognitive symptoms. A mixed-effects model for repeated measures (MMRM) was used to impute missing values.

Results. Of the 1012 patients in the ITT population, 174 (placebo $=71$; cariprazine $=103$ ) had a PANSS Cognitive subscale score of 15 or greater at baseline. At week 3, the cariprazine group demonstrated significantly greater mean improvement than the placebo group on PANSS cognitive subscale scores in both the ITT population $(-2.2 \mathrm{vs}-1.3 ; \mathrm{P}<.0001)$ and the subgroup with baseline cognitive symptoms $(-4.0 \mathrm{vs}-1.9 ; \mathrm{P}=.0002)$. In patients with baseline cognitive symptoms, improvement was significantly greater for cariprazine- versus placebo-treated patients on YMRS total score $(-16.7$ vs $-8.2 ; \mathrm{P}<.0001)$ and the individual PANSS cognitive subscale items of conceptual disorganization $(-1.1$ vs $-0.5 ; \mathrm{P}=.0004)$, difficulty in abstract thinking $(-0.8$ vs -0.3 ; $\mathrm{P}=.0044)$, stereotyped thinking $(-0.3$ vs $-0.1 ; \mathrm{P}=.0350)$, and poor attention $(-1.1 \mathrm{vs}-0.6 ; \mathrm{P}=.0043)$.
\end{abstract}

Michael E. Thase, $\mathrm{MD}^{1}$, Amanda Harrington, $\mathrm{PhD}^{2}$, Joseph Calabrese, $\mathrm{MD}^{3}$, Stuart Montgomery, $\mathrm{MD}^{4}$, Xiaomeng Niu, $\mathrm{PhD}^{5}$ and Mehul Patel, PharmD ${ }^{6}$

${ }^{1}$ University of Pennsylvania, Perelman School of Medicine, Philadelphia, PA, USA, ${ }^{2}$ AbbVie, Irvine, CA, USA, ${ }^{3}$ University Hospitals Cleveland Medical Center, Cleveland, OH, USA, ${ }^{4}$ Imperial College, London, United Kingdom, and ${ }^{5}$ AbbVie, Madison, $\mathrm{NJ}, \mathrm{USA}$

Presenting Author: Michael E. Thase 
"severely ill", and 40 or greater for "extremely ill". Area under the curve (AUC) values for these cutoffs ranged from 0.930 to 0.997 , representing outstanding sensitivity and specificity.

Conclusions. Utilizing data from 3 recent clinical trials of subjects with bipolar depression, we were able to identify MADRS severity thresholds. These empirical findings may help clinicians to understand and contextualize MADRS results from bipolar clinical research and apply to their patients in practice.

Funding. AbbVie Inc. 